\title{
Multihop Diversity for Fading Mitigation in Multihop Wireless Networks
}

\author{
Chen Dong, Lie-Liang Yang, and Lajos Hanzo \\ School of ECS, University of Southampton, SO17 1BJ, United Kingdom \\ Email: cd2g09,1ly,lh@ecs.soton.ac.uk, http://www-mobile.ecs.soton.ac.uk
}

\begin{abstract}
The concept of multihop diversity is proposed, where all the nodes of a multihop link are assumed to have buffers for temporarily storing their received packets. During each time-slot, the best hop having, for example, the highest signal-to-noise ratio (SNR), is selected from the set of those hops that have packets awaiting transmission in the buffer. The packet is then transmitted over the best hop. This hop-selection procedure yields selection diversity, but it requires the global channel knowledge of the hops' channel quality. In this paper, we assume having perfect channel knowledge and focus our attention on the principles and performance bounds of the error probability and outage probability. Our studies show that relying on multiple hops has the potential of providing a significant diversity gain, which may be exploited for enhancing the reliability of wireless multihop communications.
\end{abstract}

\section{INTRODUCTION}

Owing to their advantages, multihop communications [1-4] have drawn a lot of attention and have been investigated from different perspectives in recent years. However, in literature, it has typically been assumed that information is transmitted from a source node to the destination node one node by one node successively without any store-and-wait stage at the intermediate relay nodes [2-4]. For convenience of description, we refer to this scheme as the conventional multihop transmission scheme in our forthcoming discourse. In this conventional multihop scheme, information is transmitted over a hop during its scheduled time-slot regardless of its link quality quantified, for example, by its SNR. Hence, the overall reliability of a multihop link is dominated by that of the weakest hop and a route outage occurs, once an outage occurs in any of the invoked hops. As a result, the route error/outage performance of a multihop link usually degrades, as the number of hops increases. In order to improve the performance of multihop links, recently, novel signaling schemes have been proposed [1, $5,6]$, which require the nodes to have a store-and-wait capability. For example, in [5,6], adaptive modulation and coding (AMC) combined with automatic repeat request (ARQ) schemes has been invoked in cooperative decode-and-forward (DF) communications. Very recently, the authors in [1] have employed AMC for dual-hop cooperative communications relying on a regenerative relay node, where the AMC mode of both the hops may be configured independently.

In this contribution, we view the independently fading multiple hops of links as an adaptively configurable resource that may be exploited for achieving a diversity gain. To the best of our knowledge, the multihop diversity concept, which exploits the independent fading of communication hops for attaining diversity, has never been investigated in the open literature. The multihop diversity is achieved by assuming that every node of a multihop link has a buffer for temporarily storing the packets received. During a given time-slot, the highest-quality hop is activated from the set of hops having packets in their buffers to send, which hence results in selection diversity. Intuitively, the implementation of the proposed multihop diversity scheme requires global channel knowledge about all the hops. In this paper, however, we focus our attention on the basic principles and theoretical performance bounds under the idealized simplifying assumption that this global channel knowledge may be acquired, whenever required. Specifically, we analyze the error and outage performance of multihop links, when either buffers of limited or unlimited size are used. Furthermore, simulations are employed for study and also for verifying the accuracy of our analytical results. Note that, the terminology of multihop diversity has also been used in [7]. However, the multihop diversity considered in [7] and that defined in this paper have different meaning. In [7], it is assumed that a receiving node can receive signals from several other nodes. In this case, the multihop diversity may be obtained at the receiving node by combining the signals received from the different nodes transmitted the same information.

Our studies and performance results demonstrate that independently fading multiple hops have the potential of providing significant diversity gain for improving the reliability of communications. The error/outage performance may be improved, as the buffer size increases. However, the maximum attainable multihop diversity may be approached, provided that each of the nodes has moderate buffer size.

Naturally, once a source node completes its transmission, an increased buffering-induced delay is imposed, which is higher than that of the conventional multihop transmission scheme [8]. However, for the transmission of sufficiently large amounts of data, our multihop diversity scheme in average does not have to strike an explicit tradeoff between the delay tolerated and the achievable error/outage performance. This is because the multihop diversity scheme transmits one packet over one hop per time-slot, identically to the conventional multihop transmission scheme. The only extra time required is that of emptying the buffers at the end of a transmission block.

\section{System Model of Multihop Links}

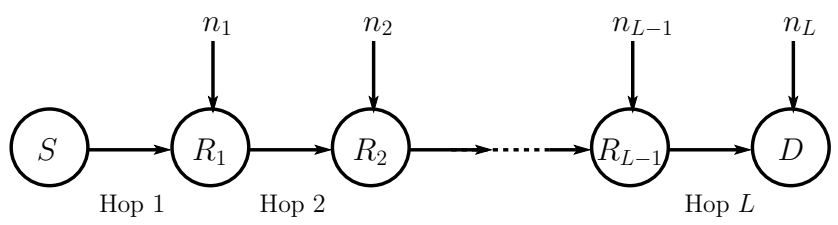

Fig. 1. System model for a multihop wireless link, where source $S$ sends message to destination $D$ via $(L-1)$ intermediate relays.

The system model under consideration is a typical multihop wireless link $[4,8]$, which is shown in Fig. 1. The multihop link consists of $(L+1)$ nodes, a source node $S$ (node 0$),(L-1)$ relay nodes $R_{1}, R_{2}, \cdots, R_{L-1}$ and a destination node $D$ (node $L$ ). The source node $S$ sends information to the destination node $D$ via $L$ hops with the aid of the $(L-1)$ relay nodes. At the relay nodes, the classic decode-and-forward (DF) protocol is employed for relaying the signals. For convenience, we denote the signal transmitted by the source node $S$ of node 0 by $x_{0}$ and its estimate at the destination node $D$ of node $L$ by $x_{L}=\hat{x}_{0}$, while the signal estimated at the $l$ th relay node by $x_{l}, l=1, \ldots, L-1$. When operated at packet level, they are correspondingly represented by $\boldsymbol{x}_{0}, \boldsymbol{x}_{l}, l=1, \ldots, K-1$, and $x_{L}=\hat{x}_{0}$. In this paper, we assume that the signals are transmitted on the basis of time-slots having a duration of $T$ seconds. The channels of the $L$ hops are assumed to experience block-based flat Rayleigh fading, where the complex-valued fading envelop of a hop remains constant within a time-slot but is independently faded for different time-slots. Based on the above assumptions, when the $(l-1)$ st node transmits a packet $\boldsymbol{x}_{l-1}$, the observations received by node $l$ can be expressed as

$$
\boldsymbol{y}_{l}=h_{l} \boldsymbol{x}_{l-1}+\boldsymbol{n}_{l}, l=1,2, \ldots, L
$$

where $h_{l}$ represents the channel gain of the $l$ th hop from node $(l-1)$ to node $l$, while $\boldsymbol{n}_{l}$ is the Gaussian noise added at node $l$. The channel 
gain $h_{l}$ is complex Gaussian with zero mean and $E\left[\left|h_{l}\right|^{2}\right]=1$. The noise samples in $\boldsymbol{n}_{l}, l=1, \ldots, L$, obey the complex Gaussian distribution with zero mean and a common variance of $\sigma^{2}=1 /\left(2 \gamma_{h}\right)$ per dimension, where $\gamma_{h}$ denotes the received average SNR per hop.

In this paper, we focus on the principles of multihop diversity as well as on its best achievable error and outage performance. For this reason, some idealized simplifying assumptions are adopted, which are summarized as follows:

- Binary phase-shift keying (BPSK) basedband modulation is assumed for signal transmission. Hence, we have $\boldsymbol{x}_{l} \in\{+1,-1\}$.

- The source node always has packets to send, hence the multihop link operates in its steady state.

- The source node $S$ and destination $D$ can store an infinite number of packets, respectively. By contrast, each of the $(L-1)$ relay nodes can only store at most $B$ packets.

- The fading processes of the $L$ hops of the multihop link are independent, while the fading of a given hop remains constant within a packet duration, but it is independently faded from one packet to another.

- There is a central control unit (CCU), which evaluates and exploits the global knowledge about the channels of the $L$ hops. Based on the global channel knowledge of the $L$ hops within a given time-slot, the CCU decides which of the $L$ nodes, i.e., nodes $0,1, \ldots, L-1$, transmits and informs the corresponding receive node without a delay and without errors. Note that, although this assumption is ideal, it is however not unreasonable. For example, for a two-hop link operated in time-division duplex (TDD) mode, the relay node can act as the CCU to decide whether the source node or itself should transmit, since it has the channel knowledge of both the first and second hops. Similarly, efficient decisionmaking/sharing strategies can be designed for links with more than two hops.

- A receive node employs ideal channel state information (CSI) for carrying out coherent detection.

- The propagation pathloss and slow shadowing fading are ignored. All the $L$ hops follow the same block-based flat Rayleigh fading. Furthermore, the received average SNR per hop in the context of the $L$ hops is the same and it is denoted by $\gamma_{h}$. Note that, this assumption is in fact reasonable, even when both the propagation pathloss and the shadow fading are considered. This is because, in multihop communications, typically, power-allocation or powercontrol is used [9] to ensure that all hops have a similar SNR and achieve a similar reliability, so that the overall reliability of a multihop link is maximal [10].

Under the above assumptions, packets are transmitted over the multihop link based on the following strategy. Among those hops having at least one packet stored in the buffer awaiting transmission, the CCU first decides which is the most reliable hop according to the instantaneous SNR values. Then, one packet is transmitted over the most reliable hop using a time-slot. According to this strategy, packets are transmitted obeying the time-division principles and hence transmitting a packet from the source node $S$ to the destination node $D$ requires $L$ time-slots.

Below we analyze the lower bounds for the BER and outage probability of the multihop link under the above-mentioned assumptions in Sections III-A and III-B, while the accurate BER and outage probability are analyzed in Sections III-C and III-D.

\section{Performance Analysis}

In this section, we first derive the lower-bounds for the BER and outage probability of the multihop link shown in Fig. 1. Then, the accurate BER and outage probability are analyzed.

Based on Fig. 1 and on the operational principles of the multihop link, as described in Section II, we can now infer that, when every relay node has a buffer of size of $B$ packets, the following events may occur. Firstly, the buffer of a relay node may be empty at some instants. In this case, this relay node cannot be the transmit node, since it has no data to transmit. Secondly, the buffer of a relay node may be full at some instants. Then, This relay node cannot be the receiving node, since it cannot accept further packets. In these cases the CCU has to choose a hop for transmission from a reduced number of hops, which results in an increased BER and outage probability due to the reduced selection diversity gain. Therefore, our lower-bounds of BER and outage probability are derived by loosening the above-mentioned constraints and assuming that each relay node has an unlimited buffer size and that a node always has packets to transmit, whenever it is instructed by the CCU to transmit. By contrast, for accurate analysis of the BER and outage probability, the above-mentioned constraints will be considered in Sections III-C and III-D.

\section{A. Lower-Bound Bit Error Rate}

In order to derive the lower-bound BER, we first derive the singlehop BER, $P_{L, e}$, under the assumptions that every relay node has an infinite buffer and that a node always has packets prepared to send. Then, the lower-bound of the end-to-end BER, $P_{L, E}$, of the multihop link shown in Fig. 1 is derived. The subscript ' $L$ ' in $P_{L, e}$ and $P_{L, E}$ stands for the lower-bound.

Considering a time-slot, when the SNR is given by $\gamma$, the conditional BER of BPSK modulation is given by [11]

$$
P_{L, e}(\gamma)=Q(\sqrt{2 \gamma})
$$

where $Q(x)$ is the Gaussian Q-function, which can alternatively be defined [12] by $Q(x)=\pi^{-1} \int_{0}^{\pi / 2} \exp \left(-x^{2} /\left(2 \sin ^{2} \theta\right)\right) d \theta$. According to the operational principles of the multihop link as described in Section II, the SNR $\gamma$ in (2) is given by

$$
\gamma=\max \left\{\gamma_{1}, \gamma_{2}, \ldots, \gamma_{L}\right\}
$$

where $\gamma_{l}$ is the SNR of the $l$ th hop within the time-slot considered, which is given by $\gamma_{l}=\left|h_{l}\right|^{2} \gamma_{h}$. We can readily show that $\gamma_{l}$ has the probability density function (PDF) $f\left(\gamma_{l}\right)=\gamma_{h}^{-1} e^{-\gamma_{l} / \gamma_{h}}, l=$ $1, \ldots, L$. Furthermore, the PDF of $\gamma$ defined in (3) can be derived as [12]

$$
f(\gamma)=\frac{L}{\gamma_{h}} \exp \left(-\frac{\gamma}{\gamma_{h}}\right)\left[1-\exp \left(-\frac{\gamma}{\gamma_{h}}\right)\right]^{L-1}
$$

The average single-hop BER $P_{L, e}$ can be obtained by averaging $P_{L, e}(\gamma)$ of (2) with respect to the PDF of (4), yielding

$$
P_{L, e}=\frac{1}{2}-\frac{L}{2} \sum_{l=0}^{L-1} \frac{(-1)^{l}}{l+1}\left(\begin{array}{c}
L-1 \\
l
\end{array}\right) \sqrt{\frac{\gamma_{h}}{l+1+\gamma_{h}}}
$$

Having obtained the single-hop lower-bound BER $P_{L, e}$, the lowerbound end-to-end BER $P_{L, E}$ can be obtained with the aid of the following approach. Let $P_{L, C}(l-1)$ and $P_{L, E}(l-1)$, where we have $l=1, \ldots, L$, be the correct and erroneous detection probabilities, respectively, after the detection at the $(l-1)$ th relay. Explicitly, we have $P_{L, C}(0)=1, P_{L, E}(0)=0$, and $P_{L, C}(l-1)=1-P_{L, E}(l-1)$. Then, the correct and erroneous detection probabilities, $P_{L, C}(l)$ and $P_{L, E}(l)$, after detection at the $l$ th relay can be expressed in matrix form as

$$
\left[\begin{array}{c}
P_{L, C}(l) \\
P_{L, E}(l)
\end{array}\right]=\left[\begin{array}{cc}
1-P_{L, e} & P_{L, e} \\
P_{L, e} & 1-P_{L, e}
\end{array}\right]\left[\begin{array}{l}
P_{L, C}(l-1) \\
P_{L, E}(l-1)
\end{array}\right]
$$

Hence, the end-to-end correct and erroneous detection probabilities $P_{L, C}$ and $P_{L, E}$ can be obtained in a recursive manner as

$$
\begin{aligned}
{\left[\begin{array}{l}
P_{L, C} \\
P_{L, E}
\end{array}\right]=\left[\begin{array}{c}
P_{L, C}(L) \\
P_{L, E}(L)
\end{array}\right] } & =\left[\begin{array}{cc}
1-P_{L, e} & P_{L, e} \\
P_{L, e} & 1-P_{L, e}
\end{array}\right]\left[\begin{array}{l}
P_{L, C}(L-1) \\
P_{L, E}(L-1)
\end{array}\right] \\
& =\left[\begin{array}{cc}
1-P_{L, e} & P_{L, e} \\
P_{L, e} & 1-P_{L, e}
\end{array}\right]^{L}\left[\begin{array}{l}
P_{L, C}(0) \\
P_{L, E}(0)
\end{array}\right] \\
& =\left[\begin{array}{cc}
1-P_{L, e} & P_{L, e} \\
P_{L, e} & 1-P_{L, e}
\end{array}\right]^{L}\left[\begin{array}{l}
1 \\
0
\end{array}\right]
\end{aligned}
$$


In (7), the matrix containing $P_{L, e}$ can be decomposed with the aid of eigenanalysis [13], yielding

$$
\begin{aligned}
\boldsymbol{M} & =\left[\begin{array}{cc}
1-P_{L, e} & P_{L, e} \\
P_{L, e} & 1-P_{L, e}
\end{array}\right] \\
& =\frac{1}{\sqrt{2}}\left[\begin{array}{cc}
1 & 1 \\
1 & -1
\end{array}\right]\left[\begin{array}{cc}
1 & 0 \\
0 & 1-2 P_{L, e}
\end{array}\right] \frac{1}{\sqrt{2}}\left[\begin{array}{cc}
1 & 1 \\
1 & -1
\end{array}\right]
\end{aligned}
$$

where $\lambda_{1}=1$ and $\lambda_{2}=1-2 P_{L, e}$ are two eigenvalues of $\boldsymbol{M}$, while $\frac{1}{\sqrt{2}}\left[\begin{array}{cc}1 & 1 \\ 1 & -1\end{array}\right]$ is a corresponding orthonormal matrix. Upon substituting (8) into (7), we can readily obtain the lower-bound endto-end BER expression, which is

$$
P_{L, E}=\frac{1}{2}-\frac{1}{2}\left(1-2 P_{L, e}\right)^{L}
$$

where the single-hop lower-bound BER $P_{L, e}$ is given by (5).

From (9), we can immediately infer that

$$
P_{L, E} \approx L P_{L, e}
$$

when $P_{L, e}$ is sufficiently small. Since $P_{L, e}$ is the BER of a typical selection combining (SC) diversity scheme associated with $L$ th-order diversity, (10) implies that, provided that each of the relay nodes has some buffer, a multihop link is capable of attaining a diversity order upto $L$, in comparison to the classic multihop link, which does not rely on any buffering at the relay nodes. Note that, for relay nodes operating without buffers, we can readily show that the BER or the approximate BER of an $L$-hop link can also be expressed by (9) or (10) upon replacing $P_{L, e}$ by $P_{e}=\left(1-\sqrt{\gamma_{h} /\left(1+\gamma_{h}\right)}\right) / 2$, which is the BER of a BPSK scheme communicating over Rayleigh fading channels [11].

\section{B. Lower-Bound Outage Probability}

The outage probability is the probability of the event that the maximal SNR of the $L$ hops is lower than a pre-set threshold. When this event occurs, either no data is transmitted on the multihop link in order to guarantee the minimum required BER, or the BER becomes higher than a predicted value, if data is still transmitted. Given a threshold $\gamma_{T}$, the lower-bound outage probability is given by

$$
P_{L, O}=\int_{0}^{\gamma_{T}} f(\gamma) d \gamma
$$

When substituting (4) into this equation, we can readily obtain

$$
P_{L, O}=\left[1-\exp \left(-\frac{\gamma_{T}}{\gamma_{h}}\right)\right]^{L}
$$

which is simply the probability that each of the $L$ hops has an SNR lower than $\gamma_{T}$.

In contrast to the above multihop diversity scheme, an outage occurs in the conventional $L$-hop transmission scheme, when one out of the $L$ hops has an SNR below the threshold $\gamma_{T}$. Therefore, the outage probability can be expressed as

$$
P_{O}=1-\left[P\left(\gamma_{l}>\gamma_{T}\right)\right]^{L}=1-\left[\int_{\gamma_{T}}^{\infty} f\left(\gamma_{l}\right) d \gamma_{l}\right]^{L}
$$

Applying the PDF of $f\left(\gamma_{l}\right)$ into this equation yields

$$
P_{O}=1-\exp \left(-\frac{L \gamma_{T}}{\gamma_{h}}\right)
$$

Furthermore, it can readily be shown that we have

$$
\lim _{\gamma_{h} \rightarrow \infty} \frac{\log \left(P_{L, O}\right)}{\log \left(P_{O}\right)}=L
$$

which means that, if the SNR $\gamma_{h}$ per hop is high, the outage probability of the $L$-hop diversity scheme decreases $L$ times faster than that of the conventional $L$-hop transmission scheme. This property also explains that our proposed transmission scheme is capable of achieving an $L$ thorder diversity.

\section{Accurate Bit Error Rate}

First, the $L$-hop link is forced to choose the best one from the set of $m$ hops in order to send information, when $(L-m)$ out of the $L$ hops do not have information to transmit. This happens either when some of the transmit nodes' buffers are empty or when some of the receive nodes' buffers are full. In this case, based on (5), the BER for $m=1, \ldots, L$ is given by

$$
P_{e}(m)=\frac{1}{2}-\frac{m}{2} \sum_{l=0}^{m-1} \frac{(-1)^{l}}{l+1}\left(\begin{array}{c}
m-1 \\
l
\end{array}\right) \sqrt{\frac{\gamma_{h}}{l+1+\gamma_{h}}}
$$

Let us express $P_{m}, m=1, \ldots, L$, the probability of the event that only $m$ out of the $L$ hops can transmit. Then, the average BER of the $L$-hop link can be formulated as

$$
P_{e}=\sum_{m=1}^{L} P_{m} P_{e}(m)
$$

Hence, what we need for evaluation of $P_{e}$ is first of all the probabilities $\left\{P_{m}\right\}$, which can be derived by treating the packet transmissions over the $L$-hop link as a Markov process.

Let us assume that the buffer size of every relay node is $B$ packets. Let the number of packets that the relays $R_{1}, R_{2}, \ldots, R_{L-1}$ hold be $b_{1}, b_{2}, \ldots, b_{L-1}$, where $b_{l}=0,1, \ldots, B$. Then, the states of the $L$ hop link can be defined in terms of the number of packets stored in the buffers of the $(L-1)$ relay nodes as

$$
S_{i}=\left[b_{1}^{(i)}, b_{2}^{(i)}, \cdots, b_{L-1}^{(i)}\right]^{T}, i=0,1, \ldots, N-1
$$

where $b_{l}^{(i)}$ denotes the number of packets held by the $l$ th relay, when the $L$-hop link is at state $i, N=(B+1)^{L-1}$ is the total number of states, which are collected into a set $\mathcal{S}=\left\{S_{0}, S_{1}, \ldots, S_{N-1}\right\}$. The state transition matrix $T$ can be derived by the following algorithm:

1) The $(B+1)^{L-1} \times(B+1)^{L-1}$ matrix $\boldsymbol{T}$ is first initialized with zero elements.

2) For row $i, i=0,1, \ldots,(B+1)^{L-1}-1$, which corresponds to the $i$ th state $S_{i}=\left[b_{1}^{(i)}, b_{2}^{(i)}, \cdots, b_{L-1}^{(i)}\right]^{T}$, the following operations are executed:

- If $b_{1}^{(i)}+1 \leq B$, the column corresponding to the output state $\left[b_{1}^{(i)}+1, b_{2}^{(i)}, \cdots, b_{L-1}^{(i)}\right]^{T}$ is set to one;

- For $l=1,2, \ldots, L-2$, if $b_{l}^{(i)}-1 \geq 0$ and $b_{l+1}^{(i)}+$ $1 \leq B$, the column corresponding to the output state $\left[b_{1}^{(i)}, \ldots, b_{l}^{(i)}-1, b_{l+1}^{i}+1, \cdots, b_{L-1}^{(i)}\right]^{T}$ is set to one;

- If $b_{L-1}^{(i)}-1 \geq 0$, the column corresponding to the output state $\left[b_{1}^{(i)}, b_{2}^{(i)}, \cdots, b_{L-1}^{(i)}-1\right]^{T}$ is set to one.

3) Each of the rows is divided by the number of ones in the row.

The state transition matrix $\boldsymbol{T}$ has the following properties:

- Matrix $T$ is a sparse matrix. Every row has at most $L$ number of nonzero elements, while the other at least $(B+1)^{L-1}-L$ elements are zero elements;

- The sum of the probabilities in each row is one;

- The number of nonzero elements in a row represents the number of hops that may be chosen by the CCU for transmission.

Having obtained the state transition matrix $T$, the steady-state probabilities can be computed by the formula [14]

$$
\boldsymbol{\pi}=\boldsymbol{T}^{T} \boldsymbol{\pi}
$$

where $\pi=\left[\pi_{0}, \pi_{1}, \ldots, \pi_{(B+1)^{L-1}-1}\right]^{T}$, and $\pi_{i}$ is the steady-state probability that the $L$-hop link is at state $S_{i}$. Equation (19) shows that $\pi$ is the right eigenvector of matrix $T^{T}$ corresponding to an eigenvalue one. Therefore, $\boldsymbol{\pi}$ can be derived with the aid of classic methods derived for solving the eigenvector problem [15]. 
From $\pi$, we can compute the probability $P_{m}, m=1,2, \ldots, L$, by adding those entries in $\pi$, which correspond to the specific rows of $T$ that have $m$ nonzero entries. Finally, given $\left\{P_{m}\right\}$, the accurate (or steady-state) BER of the $L$-hop link can be computed using (17).

\section{Accurate Outage Probability}

From the derivation of (12), we can infer that, when $m$ out of the $L$ hops are available for transmission, the conditional outage probability is given by

$$
P_{O}(m)=\left[1-\exp \left(-\frac{\gamma_{T}}{\gamma_{h}}\right)\right]^{m}, m=1,2, \ldots, L
$$

The accurate (or steady-state) outage probability of the $L$-hop link can be expressed as

$$
P_{O}=\sum_{m=1}^{L} P_{m} P_{O}(m)
$$

where $\left\{P_{m}\right\}$ are the probabilities derived in Section III-C.

\section{Performance Results}

In this section, we provide numerical and/or simulation results for both the BER and outage probability of multihop links, in order to illustrate the effect of buffer size of relay nodes on the achievable multihop diversity gain. Note that the lower bound end-to-end BER and lower-bound outage probability of multihop links may be conveniently evaluated from the formulas of (9) and (12), respectively. However, since the state transition matrix $T$ has $(B+1)^{L-1} \times(B+1)^{L-1}$ elements, the evaluation of the accurate end-to-end BER and the accurate outage probability becomes extremely hard even for a moderate value of $B$, when the scenarios of $L=4$ and a high number of hops are considered. Hence, for these cases we used solely simulations to obtain the corresponding results.

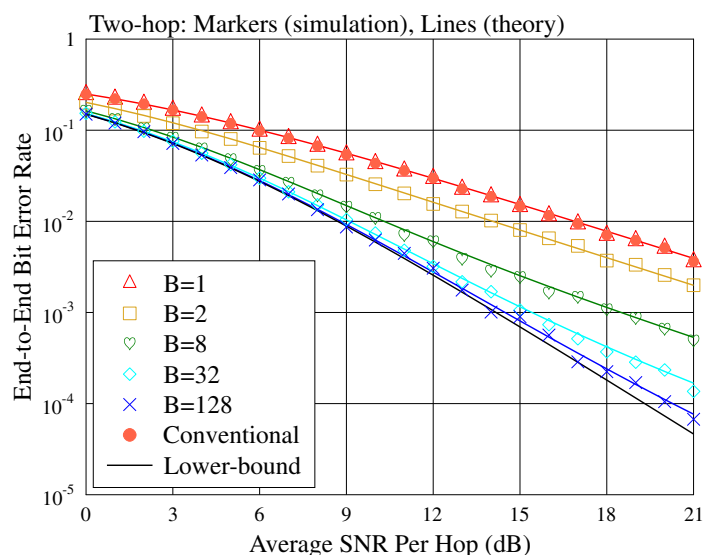

Fig. 2. BER versus average SNR per hop performance of two-hop links with relays having various buffer size, when communicating over Rayleigh fading channels.

The first set of results characterizes the relationship between the end-to-end BER performance and the buffer size of relay nodes. The results are plotted versus average SNR per hop, which is actually the average SNR per bit per hop for BPSK. In Figs. 2, 3 and 4 multihop scenarios having two, four and eight hops are characterized, respectively. In all these figures, the corresponding lower-bound end-to-end BER is provided. Furthermore, for comparison, the corresponding endto-end BER of the conventional multihop transmission scheme $[4,8]$ is also shown in Figs. 2, 3 and 4. Note that, for the two-hop link considered in Fig. 2, the end-to-end BER of the conventional scheme is the same as that of the multihop diversity scheme marked with ' $B=1$ '. From the results shown in these figures, we may draw the following observations. Firstly, multihop diversity is attainable

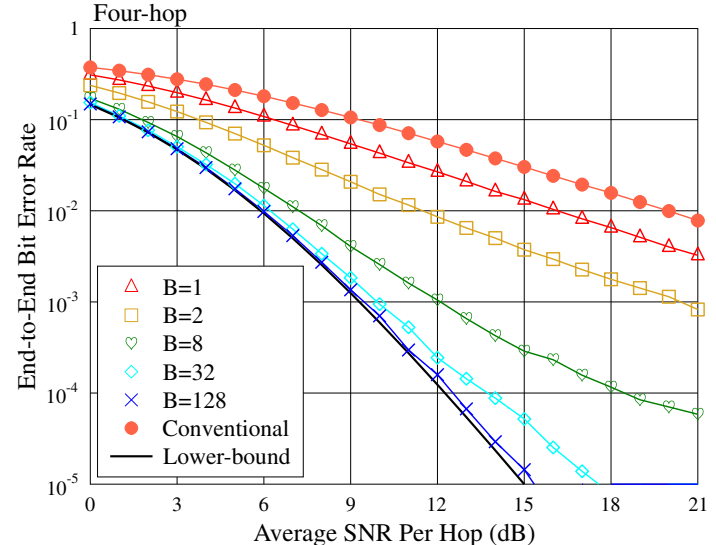

Fig. 3. BER versus average SNR per hop performance of four-hop links with relays having various buffer size, when communicating over Rayleigh fading channels.

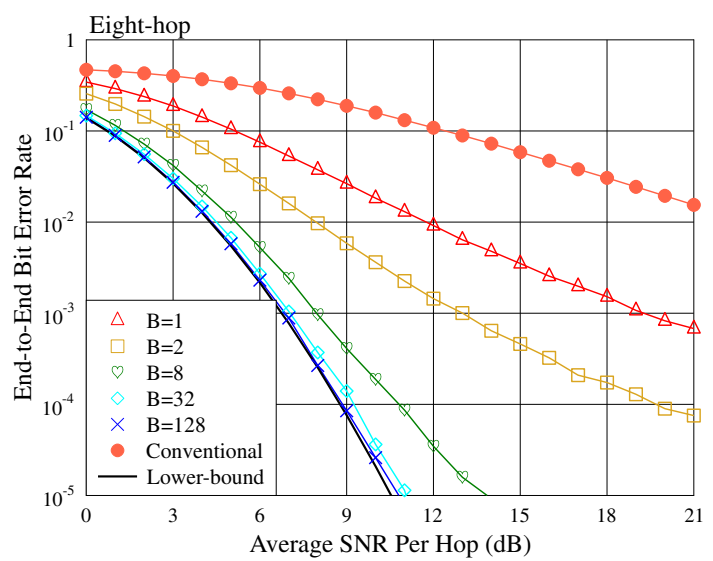

Fig. 4. BER versus average SNR per hop performance of eight-hop links with relays having various buffer size, when communicating over Rayleigh fading channels.

by equipping each of the relay nodes (also the source node that was assumed to have an infinite buffer.) with a buffer. As expected, the diversity gain improves, as the buffer size of relay nodes increases, until reaching the lower-bound end-to-end BER corresponding to infinite buffers. Secondly, the lower-bound end-to-end BER may be approached by employing buffers of reasonable size, which depends on the actual SNR. Typically, for the two-, four- and eight-hop links considered, using the buffers of size $B=32$ or 128 packets is capable of achieving most of the multihop diversity available. Thirdly, even when the relay nodes employ buffers of small size, the achievable multihop diversity may still be significant. Furthermore, for the fourand eight-hop links, multihop diversity is attainable, even when each of the buffers can only store a single packet, i.e., for $B=1$, as seen in Figs. 3 and 4. In Fig. 3, when $B=1$, the multihop diversity scheme proposed in this paper is capable of yielding an approximately $4 \mathrm{~dB}$ performance gain in comparison to the conventional multihop transmission scheme. Observe in Fig. 4 , for $B=1$, that the multihop diversity scheme may obtain in excess of $11 \mathrm{~dB}$ of SNR gain over the conventional multihop transmission scheme.

The reason for the multihop diversity scheme's ability to significantly outperform the conventional scheme is explicit. In the multihop diversity scheme proposed in this paper, the end-to-end BER performance improves as the number of hops increases owing to the increased multihop diversity. By contrast, in the conventional multihop transmission scheme $[4,8]$, the end-to-end BER performance degrades as the number of hops increases, since the packets are transmitted from 
one node to another successively.

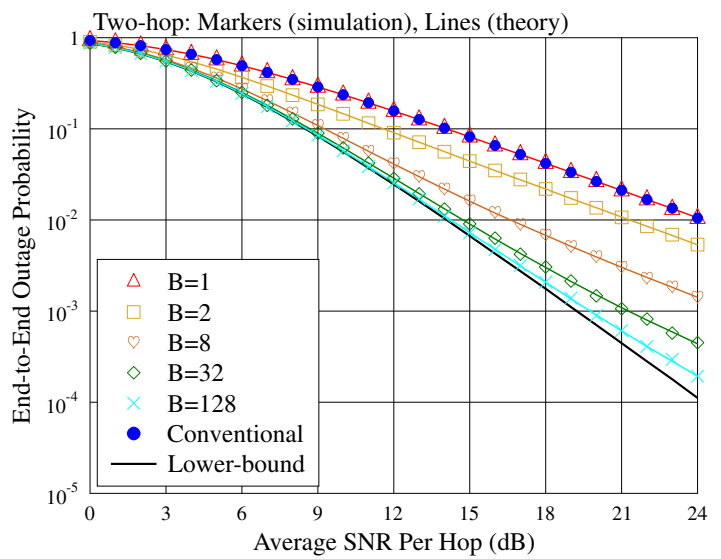

Fig. 5. Outage probability versus average SNR per hop performance of twohop links with relays having various buffer size, when communicating over Rayleigh fading channels.

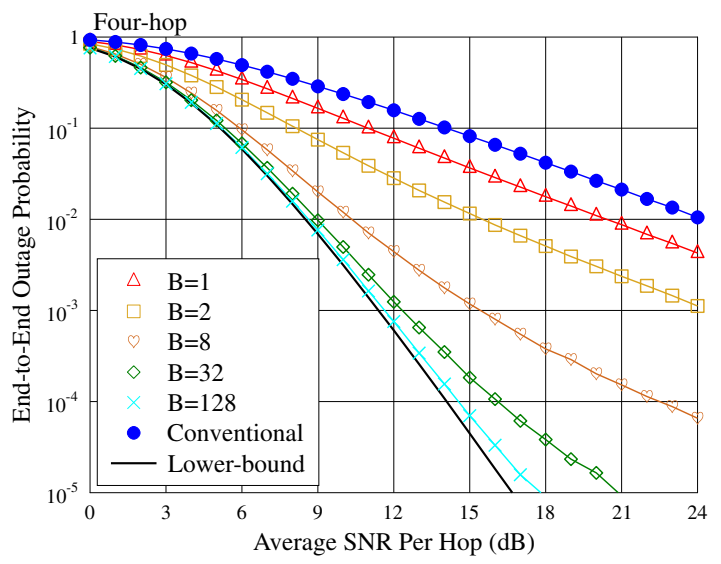

Fig. 6. Outage probability versus average SNR per hop performance of fourhop links with relays having various buffer size, when communicating over Rayleigh fading channels.

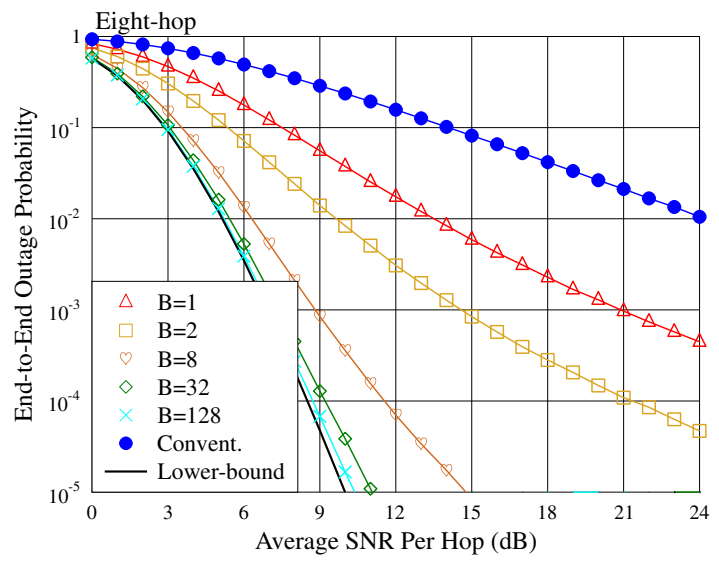

Fig. 7. Outage probability versus average SNR per hop performance of eighthop links with relays having various buffer size, when communicating over Rayleigh fading channels.

The second set of results characterizes the outage probability performance of multihop links, as shown in Figs. 5, 6 and 7 for the two-, four- and eight-hop links, respectively, when relay nodes having various buffer size are considered. Note that, in our numerical computations and simulations, the threshold $\gamma_{T}$ was adjusted to maintain a BER of 0.01 for a single-hop link. The corresponding lower-bound outage probability evaluated using (12) for the two-, four- and eight-hop links, respectively, are shown in Figs. 5, 6 and 7, while in Fig. 5, the accurate outage probability evaluated by (21) is depicted. Furthermore, the corresponding outage probability of the conventional multihop links is also provided for comparison. From these results, we can draw similar observations, as those drawn from Figs. 2, 3 and 4. A significant multihop diversity gain is attainable, when the relay nodes employ buffers of a sufficiently high size. Hence, the multihop diversity transmission scheme proposed in this contribution outperforms the conventional multihop transmission scheme $[4,8]$.

\section{CONCLUSions}

In this contribution, we have proposed and investigated a multihop diversity scheme. The BER and outage probability of multihop links have been analyzed and a range of formulas have been obtained, when assuming that BPSK signals are transmitted over all the hops, which experience iid Rayleigh fading. Our analysis and performance results show that exploiting the resource of multiple hops results in a significant diversity gain. The multihop diversity scheme significantly outperforms the conventional multihop transmission arrangement in terms of the BER/outage performance, when sufficiently large buffers are considered. Furthermore, the maximum multihop diversity can usually be approached, when each relay node has a buffer of moderate size.

\section{REFERENCES}

[1] A. Muller and H.-C. Yang, "Dual-hop adaptive packet transmission systems with regenerative relaying," IEEE Trans. on Wireless Commun. vol. 9 , no. 1 , pp. $234-244$, Jan. 2010

[2] V. Asghari, A. Maaref, and S. Aissa, "Symbol error probability analysis for multihop relaying over Nakagami fading channels," in IEEE WCNC'2010. IEEE, Apr. 2010, pp. 1-6.

[3] M. O. Hasna and M. S. Alouini, "Outage probability of multihop transmission over Nakagami fading channels,' IEEE Commun. Letters, vol. 7, no. 5 , pp. $216-218$, May 2003

[4] J. Cao, L.-L. Yang, and Z. Zhong, "Performance of multihop wireless links over generalized- $k$ fading channels," in IEEE Veh. Tech. Conf. Fall (VTC 2010-Fall). IEEE, Sept. 2010, pp. 1 - 5.

[5] Z. Lin, E. Erkip, and M. Ghosh, "Rate adaption for cooperative systems," in Proc. of IEEE GLOBECOM'06. IEEE, Nov. 2006, pp. 1-5.

[6] M. Mardani, J. Harsini, F. Lahouti, and B. Eliasi, "Joint adaptive modulation-coding and cooperative ARQ for wireless networks," in Proc. IEEE Int. Symp. Wireless Commun. Systems. IEEE, October 2008.

[7] J. Boyer, D. D. Falconer, and H. Yanikomeroglu, "Multihop diversity in wireless relaying channels," IEEE Trans. on Commun., vol. 52, no. 10, pp. $1820-1830$, Oct. 2004

[8] L. Yang, M. O. Hasna, and M. S. Alouini, "Average outage duration of multihop communication systems with regenerative relays," IEEE Trans. on Wireless Commun., vol. 4, no. 4, pp. 1366 - 1371, July 2005.

[9] B. Alawieh, Y. Zhang, C. Assi, and H. Mouftah, "Improving spatial reuse in multihop wireless networks - a survey," IEEE Commun. Survey \& Tutorials, vol. 11, no. 3, pp. $71-91,2009$.

[10] T. Liu, C. Yang, and L.-L. Yang, "Joint transmitter-receiver frequencydomain equalization in generalized multicarrier code-division multiplexing systems," IEEE Trans. on Veh. Tech., vol. 59, no. 8, pp. 3786 - 3797 , Oct. 2010.

[11] J. G. Proakis, Digital Communications, 3rd ed. McGraw Hill, 1995.

[12] M. K. Simon and M.-S. Alouini, Digital Communication over Fading Channels: A Unified Approach to Performance Analysis. New York: John Wiley \& Sons, 2000.

[13] G. Golub and C. Loan, Matrix Computations, 3rd ed. Baltimore and London: The Johns Hopkins University Press, 1996.

[14] D. Bertsekas and R. Gallager, Data Networks, 2nd ed. Englewood Cliffs, N.J. : Prentice Hall, 1992.

[15] R. Horn and C. Johnson, Matrix Analysis. Cambridge University Press, 1986. 